\title{
Post-resuscitation care
}

Sohil Pothiawala, FAMS, MRCSEd

\begin{abstract}
Following return of spontaneous circulation (ROSC) after cardiac arrest, the challenge is to institute measures that ensure a higher likelihood of neurologically intact survival. Regardless of the cause of collapse, multiple organ systems may be affected secondary to post-cardiac arrest syndrome. Interventions required for post-ROSC care are bundled into a care regimen: prompt identification and treatment of the cause of cardiac arrest; and treatment of electrolyte abnormalities. It is also essential to establish definitive airway management to maintain normocapnic ventilation, prevent hyperoxia, and optimise haemodynamic management via judicious intravenous fluids and vasoactive drugs. Targeted temperature management after ROSC confers neuroprotection and leads to improved neurological outcomes. Glycaemic control of blood glucose levels at $6-10 \mathrm{mmol} / \mathrm{L}$, adequate seizure management and measures to optimise neurological functions should be integrated into the care bundle. The interventions outlined can potentially lead to more patients being discharged from hospital alive with good neurological function.
\end{abstract}

Keywords: airway management, care bundle, hyperoxia, neurological function, post-resuscitation

\section{INTRODUCTION}

The primary goal of resuscitation of a cardiac arrest patient using basic and advanced life support measures is to achieve return of spontaneous circulation (ROSC). Despite various advances in resuscitation science, the overall percentage of patients who attain ROSC (on-scene or in a hospital emergency department) and are eventually discharged from hospital alive remains low. This article highlights the interventions that can be incorporated as a bundle of post-ROSC interventions, with an aim to narrow the gap between ROSC and neurologically intact survival in patients who sustain cardiac arrest.

\section{PATHOPHYSIOLOGY OF POST-CARDIAC ARREST SYNDROME}

Regardless of the cause of cardiac arrest, the hypoxia, ischaemia and reperfusion that occur during and after the resuscitation phase result in damage to multiple organ systems. This condition, called post-cardiac arrest syndrome, comprises four major components:

(a) Persistent precipitating pathology: the precipitating aetiology that leads to cardiac arrest needs to be rapidly identified and addressed. The most common pathology is coronary thrombus, which causes myocardial infarction. Other noncoronary causes that can lead to cardiac arrest include hypoxia, pulmonary embolism (PE) and sepsis.

(b) Anoxic brain injury: the reperfusion that occurs after a period of cerebral hypoxia results in the formation of free radicals and activation of cell-death signalling pathways causing disturbance of cerebral microvascular homeostasis. This injury can continue for hours to days and is aggravated by additional insults such as fever, poor glucose control and hyperoxia. The symptoms of anoxic brain injury include coma, seizures, myoclonus, various degrees of neurocognitive dysfunction and brain death.

(c) Post-cardiac arrest myocardial dysfunction: there is hypokinesia of the cardiac muscles associated with a significant drop in left ventricular ejection fraction, especially during the first 24-48 hours after ROSC. It occurs despite preserved coronary blood flow. This manifests as tachycardia, hypotension, poor cardiac output and elevated left ventricular end-diastolic pressure.

(d) Systemic ischaemia/reperfusion response: whole-body hypoxia/ischaemia followed by post-ROSC reperfusion results in systemic inflammation, endothelial activation, and activation of the immunologic and coagulation pathways. This resembles the pathophysiology occurring during severe sepsis and increases the risk of multiple organ dysfunction syndrome. Clinical manifestations include fever and altered oxygen consumption, as well as increased susceptibility to infection.

Post-resuscitation care is an evolving science that requires a well-coordinated multidisciplinary effort with participation of physicians from the disciplines of emergency medicine, critical and intensive care, nursing, cardiology and anaesthesiology, at the very least. There is a need to identify approaches and optimise workflow for the integration of post-ROSC care as a standard of care in treating cardiac arrest patients who attain ROSC. This can be achieved by determining how the various interventions needed in post-resuscitation care can be bundled into a care regimen and implemented in most clinical care areas. In this article, the National Resuscitation Council, Singapore, has evaluated the current research in this field and produced the guidelines that can be further adopted in our clinical practice and updated with research in our local setting and environment.

\section{SCOPE OF POST-ROSC CARE}

Cardiac arrest is multifactorial and can severely affect multiple organ systems regardless of cause, hypoxaemia, ischaemic injuries and reperfusion injuries. ${ }^{(1)}$ Post-resuscitation care must be tailored 
to the needs of the individual patient. The following areas need to be addressed to improve outcomes: (a) identification and treatment of the cause of cardiac arrest; (b) airway and ventilation management; (c) haemodynamic management; (d) targeted temperature management (TTM) or therapeutic hypothermia (TH); (e) glycaemic control; and (f) seizure management and neuroprognostication.

\section{Identification and treatment of the cause of cardiac arrest} Once ROSC has been achieved, the factors that contributed to cardiac arrest should be identified early for appropriate intervention to treat the cause. A good history of the events leading to the collapse, careful physical examination and basic investigation would help to rapidly determine the cause. Some of these causes might become apparent during resuscitation of the patient, especially while evaluating the $5 \mathrm{Hs}$ and $5 \mathrm{Ts}$ of cardiac arrest. Some common known causes are listed below.

\section{Coronary artery disease}

As soon as possible following ROSC, 12-lead electrocardiography (ECG) should be performed to diagnose ST-segment elevation myocardial infarction (STEMI), and an immediate coronary angiography arranged. In a series of patients who underwent urgent coronary angiography following cardiac arrest, a coronary artery lesion was found in $96 \%$ of the patients with STEMI and in 58\% of the patients without ST elevation on ECG. ${ }^{(2)}$ Myocardial and neurological functions can improve after percutaneous coronary intervention following cardiac arrest. ${ }^{(3)}$ Multiple studies have demonstrated improved survival to hospital discharge, as well as improved neurologically favourable outcomes associated with emergency coronary angiography in patients with ST elevation after cardiac arrest. Some patients are hypotensive despite revascularisation and may need mechanical augmentation via intraaortic balloon pump or extracorporeal membrane oxygenation.

Thus, immediate coronary angiography should be performed on out-of-hospital cardiac arrest patients with suspected cardiac aetiology and STEMI on ECG following ROSC (Class I). It is also reasonable to perform emergency coronary angiography following ROSC in unstable patients with suspected cardiac aetiology but no ST elevation on ECG (Class Ila). All patients should undergo continuous cardiac monitoring for arrhythmias in the period following cardiac arrest. Arrhythmias should be managed appropriately as per resuscitation guidelines.

\section{Acute pulmonary embolism}

Cardiac arrest due to PE is often not obvious and accounts for about $2 \%-10 \%$ of cases. Indicators of this cause include poor arterial oxygen saturation following ROSC with appropriate ECG changes. Current guidelines do not support the routine use of fibrinolytics during cardiac arrest. Following ROSC, it is preferable to confirm the diagnosis of PE on imaging before fibrinolysis is initiated. When direct imaging is unavailable or unsafe because of the patient's unstable condition, fibrinolytics may be used in post-cardiac arrest patients who are suspected to have collapsed from severe PE, i.e. patients with sustained hypotension (systolic blood pressure $<90 \mathrm{~mm} \mathrm{Hg}$ for at least 15 minutes or requiring inotropic support, not clearly due to a cause other than PE), a high clinical pretest probability of PE and right ventricular dysfunction on bedside transthoracic echocardiography.

\section{Cardiotoxic agents}

Drugs such as tricyclic antidepressants, cardiac glycosides and recreational drugs are the main pharmacologic agents that result in cardiac arrest. However, identifying the drug involved is usually a major challenge. Since most cardiotoxic drugs are water-soluble and may be excreted by the kidney, forced alkaline diuresis may be employed. This is a slow process owing to the mechanisms of excretion of the drugs. The use of intralipids to enhance the elimination of cardiotoxic drugs may be considered once ROSC is achieved. If started before collapse, prolonged cardiopulmonary resuscitation may be required to allow time for these agents to exert their effects. These agents must be continued for at least 24 hours following ROSC. If the cardiotoxic agent is known, available antidotes may be administered. Frequently, massive drug overdoses resulting in cardiovascular collapse require rapid removal via haemodialysis. However, this may only be considered if the patient has attained ROSC and supportive care is in progress.

\section{Metabolic disturbances}

A number of metabolic disorders such as hyperkalaemia, hypokalaemia and hypercalcaemia can cause cardiac arrest. ECG following ROSC may be the only initial clue to this diagnosis. Treatment to correct this metabolic disorder, initiated during resuscitation or following ROSC, must proceed in tandem with other supportive care measures. For instance, hyperkalaemic patients would require calcium gluconate and glucose plus insulin, in addition to haemodialysis, to remove the excessive potassium load, while hypokalaemic patients would need replacement therapy.

\section{Sepsis}

Sepsis is one of the common causes of cardiovascular collapse. Once suspected, blood cultures should be obtained and the appropriate intravenous antibiotics administered, in addition to identifying the source of infection and appropriate management. Protocolised resuscitation of patients using sepsis-care bundles has been shown to decrease mortality in this group of patients. ${ }^{(6)}$

\section{Airway and ventilation management}

Awake patients who are able to maintain their airway and have spontaneous respiratory effort can be monitored without intubation. Supplemental oxygen is recommended to maintain blood oxygen saturation $\left(\mathrm{SpO}_{2}\right)$ of $94 \%-98 \%$. Following ROSC, comatose patients should have a definitive airway established and mechanical ventilation commenced. Previous guidelines recommend appropriate titration of supplemental oxygenation to prevent hypoxia and avoid prolonged periods of hyperoxia. There is increasing evidence to suggest that excessive oxidative stress during hyperoxia may harm various organs, causing neuronal damage as well as irreversible changes within the alveolar space. Severe hyperoxia was independently associated with decreased 
survival to hospital discharge. ${ }^{(7)}$ Following ROSC, hyperoxaemia during the reperfusion phase with $100 \%$ oxygen leads to increased brain lipid peroxidation, greater metabolic dysfunction and neurological degeneration. These concerns and their impact on short-term functional outcome have resulted in calls to ventilate with room air or fraction of inspired oxygen $\left(\mathrm{FiO}_{2}\right)$ titrated to maintain a pulse oximetry reading of $94 \%-98 \%{ }^{(8)}$

In the phase following ROSC, it is reasonable to commence mechanical ventilation using the highest oxygen concentration $\left(\mathrm{FiO}_{2} 100 \%\right)$ to avoid hypoxia. Once reliable $\mathrm{SpO}_{2}$ can be measured or arterial blood gas is obtained to measure oxygenation, it is important to titrate $\mathrm{FiO}_{2}$ to maintain the oxyhaemoglobin saturation at $94 \%-98 \%$ on pulse oximetry.

Hypocapnia is associated with worse neurological outcomes. ${ }^{(9)}$ It is suggested that the initial ventilator settings should begin with tidal volumes of $6-8 \mathrm{~mL} / \mathrm{kg}$ body weight and ventilatory rates of 10-12 breaths/minute. The aim of optimal ventilation should be to maintain normocarbia (end-tidal carbon dioxide $30-40 \mathrm{mmHg}$ or partial pressure of arterial carbon dioxide 35-45 mmHg). Hyperventilation is not recommended, as it decreases the partial pressure of carbon dioxide, which in turn decreases cerebral blood flow, causing cerebral vasoconstriction and aggravating anoxic brain damage. Minute ventilation should thus be titrated, guided by serial arterial blood gas measurements.

\section{Haemodynamic management}

Post-ROSC patients are often haemodynamically unstable and their management can be challenging. Although optimal haemodynamic goals remain undefined, initiatives to maintain adequate cerebral and coronary perfusion pressures as well as blood flow to other vital organs must be instituted. The main goal of haemodynamic management is to avoid hypotension and achieve a systolic blood pressure of at least $90 \mathrm{mmHg}$ or a mean arterial pressure of $65 \mathrm{mmHg}$ following resuscitation. ${ }^{(10)}$ Targets for other haemodynamic parameters (e.g. cardiac output, cardiac index, mixed/central venous oxygen saturation $\left[\mathrm{ScvO}_{2}\right]$ or urine output) are still undefined and can vary among patients, based on their specific comorbidities.

Intravenous fluids and inotropic drugs should be titrated to optimise blood pressure, cardiac output and urine output, and administered judiciously. The aim is to optimise cardiac output, tissue perfusion and oxygen delivery to achieve an $\mathrm{ScvO}_{2} \geq 70 \%$. ${ }^{(11)}$ Although there is no gold standard, pharmaceutical agents that may be used to support circulation include adrenaline, noradrenaline, dopamine and dobutamine. Dosages must be adjusted based on the parameters monitored. Echocardiography should usually be performed at 24-48 hours following ROSC to monitor ejection fraction and rule out regional wall motion abnormalities.

\section{Targeted temperature management or therapeutic hypothermia}

Hypoxic brain injury is a major cause of morbidity and mortality following resuscitation. ${ }^{(12)}$ TTM or TH following ROSC confers neuroprotection through a variety of mechanisms, including decreasing cerebral oxygen demand, reducing the cellular effects of reperfusion and decreasing the production of reactive free oxygen radicals. Thus, $\mathrm{TH}$ following ROSC has been demonstrated to improve neurological outcomes. ${ }^{(13,14)}$ New evidence demonstrating the efficacy of a broader range of temperatures $^{(15)}$ (including normothermia) and the prevention of fever has led to increasing use of the term TTM over TH.

TTM should be initiated immediately in resuscitated adult patients who are comatose at ROSC, regardless of the first recorded rhythm (shockable and nonshockable) or setting of arrest (out-of-hospital or in-hospital), with a target temperature of $33^{\circ} \mathrm{C}-36^{\circ} \mathrm{C}$; TTM should be maintained for at least 24 hours. ${ }^{(13-15)}$ Lower temperature targets are associated with increased risk of sepsis, bradydysrhythmia and coagulopathy, and should be avoided in these patients. Fever should be prevented in all patients with cardiac arrest and abnormal levels of consciousness. Thus, the maximum target temperature for cooling for all patients should not exceed $36^{\circ} \mathrm{C}$.

Temperature management may be initiated and target temperature achieved or maintained by a variety of methods. These include the use of: (a) surface cooling with ice packs or cooling blankets applied to the groin, axillae, neck and large areas of skin, or a helmet device containing aqueous glycerol solution; ${ }^{(16)}$ (b) judicious amounts of cold $\left(2{ }^{\circ} \mathrm{C}-4^{\circ} \mathrm{C}\right)$ infusions, especially normal saline or a balanced electrolyte fluid, ${ }^{(17)}$ as large volumes may be associated with an increased risk of pulmonary oedema; ${ }^{(18)}$ (c) devices that initiate evaporative transnasal cooling, ${ }^{(19)}$ and (d) endovascular cooling catheters. ${ }^{(20)}$

During the initiation of TTM and maintenance of target temperature, the core body temperature should be monitored using bladder temperature catheters, or oesophageal or central venous probes. Axillary, tympanic membrane, oral and even rectal temperatures differ from the core body temperature, and are thus unreliable. The target temperature should be maintained for 24 hours before rewarming.

Shivering, a side effect of hypothermia, has an adverse impact on the management of target temperature, and if necessary, the patient should be sedated and paralysed to reduce its undesirable effects. Neurological monitoring using electroencephalogram or bispectral index brain system is necessary during paralysis in order to detect seizures or awareness. If paralytics are needed, analgesia and sedation should be titrated, as necessary, for mechanical ventilation and comfort.

The patient should be gradually rewarmed at approximately $0.25^{\circ} \mathrm{C}-0.33^{\circ} \mathrm{C}$ per hour (not exceeding $0.5^{\circ} \mathrm{C}$ per hour) until return to normothermia. Rapid rewarming can lead to cerebral oedema, seizures and hyperkalaemia. Close monitoring of electrolytes and coagulation indices should be performed at fourhour intervals. Following rewarming, the focus should be placed on the avoidance of rebound hyperthermia, as the occurrence of hyperthermia in the first few days after cardiac arrest is associated with neurological injury and worse outcomes.

Some potential complications of TTM include increased susceptibility to infection, hypotension, coagulopathy, arrhythmia, hyperglycaemia and electrolyte imbalance. ${ }^{(16,21-23)}$ Appropriate care must be taken to prevent infection and antibiotics should be 
used, if needed. Haemodynamic support and electrolyte levels should also be monitored, and insulin therapy initiated to prevent hyperglycaemia. Patients undergoing TTM need to be managed in the intensive care unit with close monitoring of vital parameters.

\section{Glycaemic control}

Hyperglycaemia following ROSC has been associated with increased mortality and worse neurological outcomes. ${ }^{(24)}$ Similarly, hypoglycaemia is also associated with poor outcomes in critically ill patients; ${ }^{(25)}$ the optimal range of blood sugar in these patients remains unknown. Strict blood sugar control with intensive insulin therapy increases the risk of hypoglycaemia, which has been associated with increased mortality. ${ }^{(26,27)} \mathrm{A}$ study comparing strict versus moderate glucose control did not show any mortality benefit with strict monitoring in post-cardiac arrest patients. ${ }^{(27)}$ Thus, blood sugar levels should be maintained at $6-10 \mathrm{mmol} / \mathrm{L}$ through regular blood glucose monitoring and insulin therapy.

\section{Seizure management and neuroprognostication}

The prevalence of seizures in post-cardiac arrest patients is about $12 \%-20 \%$. As seizure is detrimental to brain function, it should be treated promptly with benzodiazepines and other anticonvulsant medication. There is no role for the prophylactic administration of anticonvulsant drugs. Electroencephalogram should be performed without delay, and readings should be monitored frequently or continuously in comatose patients following ROSC.

Neuroprognostication in post-cardiac arrest patients is clinically challenging. Brain injury is a result of initial ischaemic injury followed by reperfusion injury occurring during the hours or days after ROSC. Features indicating brain injury in post-ROSC patients include coma, seizures, myoclonus and various degrees of neurocognitive dysfunction, ranging from memory deficits to a persistent vegetative state and, finally, brain death.

The earliest time for prognostication in post-ROSC patients treated with TTM is 72 hours after return to normothermia. In patients who were not treated with TTM, prognostication should be performed 72 hours after cardiac arrest. As such, decisions on a do-not-resuscitate order or withdrawal of care should be avoided for 72 hours following ROSC. However, in cases where patients have an underlying terminal disease, brain herniation or other non-survivable situations, withdrawal of care may be considered before 72 hours.

\section{CONCLUSION}

Management of cardiac arrest patients following ROSC is complex and requires a multidisciplinary approach. All hospitals should establish standardised protocols for the initiation and management of bundled post-ROSC care, which may eventually lead to improved patient outcomes.

\section{REFERENCES}

1. Neumar RW, Nolan JP, Adrie C, et al. Post-cardiac arrest syndrome: epidemiology, pathophysiology, treatment, and prognostication. A consensus statement from the International Liaison Committee on Resuscitation. Circulation 2008; 118:2452-83.

2. Dumas F, Cariou A, Manzo-Silberman S, et al. Immediate percutaneous coronary intervention is associated with better survival after out-of-hospital cardiac arrest: insights from the PROCAT (Parisian Region Out of Hospital Cardiac ArresT) registry. Circ Cardiovasc Interv 2010; 3:200-7.

3. Laurent I, Monchi M, Chiche JD, et al. Reversible myocardial dysfunction in survivors of out-of-hospital cardiac arrest. J Am Coll Cardiol 2002; 40:2110-6.

4. Kürkciyan I, Meron G, Sterz F, et al. Pulmonary embolism as a cause of cardiac arrest: presentation and outcome. Arch Intern Med 2000; 160:1529-35.

5. Callaway CW, Soar J, Aibiki M, et al; Advanced Life Support Chapter Collaborators. Part 4: Advanced Life Support: 2015 International Consensus on Cardiopulmonary Resuscitation and Emergency Cardiovascular Care Science With Treatment Recommendations. Circulation 2015; 132(16 Suppl 1):S84-145.

6. Dellinger RP, Levy MM, Rhodes A, et al; Surviving Sepsis Campaign Guidelines Committee including the Pediatric Subgroup. Surviving sepsis campaign: international guidelines for management of severe sepsis and septic shock: 2012. Crit Care Med 2013; 41:580-637.

7. Elmer J, Scutella M, Pullalarevu J, et al; Pittsburgh Post-Cardiac Arrest Service (PCAS). The association between hyperoxia and patient outcomes after cardiac arrest: analysis of a high-resolution database. Intensive Care Med 2015; 41:49-57.

8. Liu Y, Rosenthal RE, Haywood Y, et al. Normoxic ventilation after cardiac arrest reduces oxidation of brain lipids and improves neurological outcome. Stroke 1998; 29:1679-86.

9. Roberts BW, Kilgannon JH, Chansky ME, et al. Association between postresuscitation partial pressure of arterial carbon dioxide and neurological outcome in patients with post-cardiac arrest syndrome. Circulation 2013; 127:2107-13.

10. Bray JE, Bernard S, Cantwell K, Stephenson M, Smith K; VACAR Steering Committee. The association between systolic blood pressure on arrival at hospital and outcome in adults surviving from out-of-hospital cardiac arrests of presumed cardiac aetiology. Resuscitation 2014; 85:509-15.

11. Gaieski DF, Band RA, Abella BS, et al. Early goal-directed hemodynamic optimization combined with therapeutic hypothermia in comatose survivors of out-of-hospital cardiac arrest. Resuscitation 2009; 80:418-24.

12. Laver S, Farrow C, Turner D, Nolan J. Mode of death after admission to an intensive care unit following cardiac arrest. Intensive Care Med 2004; 30:2126-8.

13. Hypothermia after Cardiac Arrest Study Group. Mild therapeutic hypothermia to improve the neurologic outcome after cardiac arrest. N Engl J Med 2002; 346:549-56.

14. Bernard SA, Gray TW, Buist MD, et al. Treatment of comatose survivors of out-of-hospital cardiac arrest with induced hypothermia. N Engl J Med 2002; 346:557-63

15. Nielsen N, Wetterslev J, Cronberg T, et al; TTM Trial Investigators. Targeted temperature management at $33^{\circ} \mathrm{C}$ versus $36^{\circ} \mathrm{C}$ after cardiac arrest. N Engl J Med 2013; 369:2197-206.

16. Jarrah S, Dziodzio J, Lord C, et al. Surface cooling after cardiac arrest: effectiveness, skin safety, and adverse events in routine clinical practice. Neurocrit Care 2011; 14:382-8.

17. Kim F, Olsufka $M$, Carlbom D, et al. Pilot study of rapid infusion of $2 \mathrm{~L}$ of 4 degrees $\mathrm{C}$ normal saline for induction of mild hypothermia in hospitalized, comatose survivors of out-of-hospital cardiac arrest. Circulation 2005; 112:715-9.

18. Kim F, Nichol G, Maynard C, et al. Effect of prehospital induction of mild hypothermia on survival and neurological status among adults with cardiac arrest: a randomized clinical trial. JAMA 2014; 311:45-52.

19. Castrén M, Nordberg P, Svensson L, et al. Intra-arrest tranasal evaporative cooling: a randomized, prehspital, multicentre study (PRINCE: Pre-ROSC IntraNasal Cooling Effectiveness). Circulation 2010; 122:729-36.

20. Al-Senani FM, Graffagnino C, Grotta JC, et al. A prospective multicenter pilot study to evaluate the feasibility and safety of using the CoolGard System and Icy catheter following cardiac arrest. Resuscitation 2004; 62:143-50.

21. Bro-Jeppesen J, Hassager C, Wanscher M, at al. Post-hypothermia fever is associated with increased mortality after out-of-hospital cardiac arrest. Resuscitation 2013; 84:1734-40.

22. Perbet S, Mongardon N, Dumas F, et al. Early-onset pneumonia after cardiac arrest: characteristics, risk factors and influence on prognosis. Am J Respir Crit Care Med 2011; 184:1048-54.

23. Cueni-Villoz N, Devigili A, Delodder F, et al. Increased blood glucose variability during therapeutic hypothermia and outcome after cardiac arrest. Crit Care Med 2011; 39:2225-31.

24. Kennedy A, Soar J. Management of glucose post cardiac arrest. In: BestBets Best Evidence Topics [online]. Available at: http://bestbets.org/bets/bet.php?id=1043. Accessed March 3, 2017.

25. Arabi YM, Tamim HM, Rishu AH. Hypoglycemia with intensive insulin therapy in critically ill patients: predisposing factors and association with mortality. Crit Care Med 2009; 37:2536-44.

26. Krinsley JS, Grover A. Severe hypoglycemia in critically ill patients: risk factors and outcomes. Crit Care Med 2007; 35:2262-7.

27. Oksanen T, Skrifvars MB, Varpula T, et al. Strict versus moderate glucose control after resuscitation from ventricular fibrillation. Intensive Care Med 2007; 33:2093-100. 